\title{
Recombination rates from potential models close to the unitary limit
}

\author{
E. Garrido, ${ }^{1}$ M. Gattobigio, ${ }^{2}$ and A. Kievsky ${ }^{3}$ \\ ${ }^{1}$ Instituto de Estructura de la Materia, CSIC, Serrano 123, E-28006 Madrid, Spain \\ ${ }^{2}$ Université de Nice-Sophia Antipolis, Institut Non-Linéaire de Nice, CNRS, 1361 route des Lucioles, 06560 Valbonne, France \\ ${ }^{3}$ Istituto Nazionale di Fisica Nucleare, Largo Pontecorvo 3, 56100 Pisa, Italy
}

(Received 7 June 2013; published 9 September 2013)

\begin{abstract}
We investigate universal behavior in the recombination rate of three bosons close to threshold. Using the He-He system as a reference, we solve the three-body Schrödinger equation above the dimer threshold for different potentials having large values of the two-body scattering length $a$. To this aim, we use the hyperspherical adiabatic expansion and we extract the $S$ matrix through the integral relations recently derived. The results are compared to the universal form, $\alpha \approx 67.1 \sin ^{2}\left[s_{0} \ln \left(\kappa_{*} a\right)+\gamma\right]$, for different values of $a$ and selected values of the three-body parameter $\kappa_{*}$. A good agreement with the universal formula is obtained after introducing a particular type of finiterange corrections, which have been recently proposed [A. Kievsky and M. Gattobigio, Phys. Rev. A 87, 052719 (2013)]. Furthermore, we analyze the validity of the above formula in the description of a very different system: neutron-neutron-proton recombination. Our analysis confirms the universal character of the process in systems of very different scales having a large two-body scattering length.
\end{abstract}

DOI: 10.1103/PhysRevA.88.032701

PACS number(s): 34.50.Cx, 31.15.xj, 25.45.De

\section{INTRODUCTION}

The system of three identical bosons having a large two-body scattering length has been the subject of intense investigations in recent years. As shown by Efimov in a sequence of papers [1,2], the three-body spectrum consists of an infinite number of states that accumulate to zero with the ratio between two consecutive states being $E_{3}^{n+1} / E_{3}^{n}=$ $e^{-2 \pi / s_{0}}$. In other words, in the limit $a \rightarrow \infty$, the two-body system shows a continuous scale invariance that is broken in the $s$-wave three-body sector of a bosonic system. The residual symmetry is the discrete scale invariance (DSI); namely, the physics is invariant under the rescaling $r \rightarrow \Lambda^{n} r$, where the constant is usually written $\Lambda=e^{\pi / s_{0}}$, with $s_{0} \approx 1.00624$ a universal number that characterizes a system of three identical bosons.

The DSI constrains the form of the observables to be logperiodic functions of the control parameters. One example is the atom-dimer scattering length, which has the general form

$$
a_{\mathrm{AD}} / a=d_{1}+d_{2} \tan \left[s_{0} \ln \left(a \kappa_{*}\right)+d_{3}\right],
$$

where $d_{1}, d_{2}, d_{3}$ are universal constants [3]. For atom-dimer collisions below the dimer breakup threshold, DSI imposes the following universal form for the effective-range function:

$$
k a \cot \delta=c_{1}(k a)+c_{2}(k a) \cot \left[s_{0} \ln \left(a \kappa_{*}\right)+\phi(k a)\right],
$$

with $\delta$ the atom-dimer phase shift and $c_{1}, c_{2}, \phi$ universal functions of the dimensionless variable $k a$, where $k^{2}=$ $(4 / 3) E_{i} /\left(\hbar^{2} / m\right)$, where $E_{i}$ is the incident energy in the atom-dimer center-of-mass frame and $m$ is the boson mass. It is well known that for $k \rightarrow 0$, the effective-range function satisfies the limit $k a \cot \delta \rightarrow-a / a_{\mathrm{AD}}$, which implies that at $k=0$, the constants $d_{1}, d_{2}, d_{3}$ and $c_{1}(0), c_{2}(0), \phi(0)$ are related by simple trigonometric relations. A parametrization of the universal constants and functions can be found in Ref. [3].

The DSI also constrains the form of the $S$ matrix for collisions above the dimer threshold, leading to the following peculiar form for the recombination rate at threshold [4-6]:

$$
K_{3}=\frac{128 \pi^{2}(4 \pi-3 \sqrt{3})}{\sinh ^{2}\left(\pi s_{0}\right)+\cosh ^{2}\left(\pi s_{0}\right) \cot ^{2}\left[s_{0} \ln \left(a \kappa_{*}\right)+\gamma\right]} \frac{\hbar a^{4}}{m},
$$

which, by using the large value of the factor $e^{2 \pi s_{0}} \approx 515$, can be approximated by

$$
K_{3}=\alpha a^{4} \hbar / m \approx 67.1 \sin ^{2}\left[s_{0} \ln \left(\kappa_{*} a\right)+\gamma\right] a^{4} \hbar / m,
$$

where $\gamma=1.16$ [3].

In this work, we study in detail the universal behavior of $\alpha$ by solving the Schrödinger equation for a family of attractive two-body Gaussian potentials describing the He-He system. These potentials are constructed to reproduce the two-body binding energy $E_{2}$, the two-body scattering length $a$, and the effective range $r_{s}$ of the LM2M2 potential, widely used in the literature [7]. The variation of the potential strength produces different values of the scattering length $a$, allowing a comparison of the recombination rate given by Eq. (3). The model includes a three-body interaction necessary to tune the trimer energy of the LM2M2 potential. This procedure is equivalent, up to range corrections, to the implementation of effective-field theory (EFT) at leading order (LO). This strategy has been used before in the study of the atom-dimer effective-range function $k a \cot \delta$ [8]. It has been found that the zero-range formula of Eq. (2) has to be modified in order to describe results obtained through the solution of the Schrödinger equation using finite-range potentials. The modification consists in a shift on the variable $\kappa_{*} a$, and in the replacement of $k a \cot \delta$ by $k a_{B} \cot \delta$, where $a_{B}$ is related to the two-body binding energy through $E_{2}=\hbar^{2} / m a_{B}^{2}$. In the present analysis, we find that the same type of modification has to be made in order to describe the numerical results.

The universal character of Eq. (3) allows its application to very different systems. Here we extend the study on atomic systems to describe a nuclear system: the neutronneutron-proton recombination rate close to threshold. This study is twofold. On one hand, we would like to confirm that 
systems whose energies and sizes differ by several order of magnitude are still described by the same universal equation. On the other hand, very low-energy recombination in nuclear systems can be achieved in stars, and the present study can therefore be applied to those systems in which the three-body structure is dominant, e.g., neutron-neutron-core systems. As an example, we can mention the recently performed studies in recombination of ${ }^{3} \mathrm{H}$ [9] and low-energy $n-{ }^{19} \mathrm{C}$ collisions [10].

Finally, we remark that in cold-atom physics, the search for universal behavior is a very active sector of research. At present, there is intense experimental activity to study Efimov physics in trapped ultracold gases. In these systems, the recombination rate is of major importance since it is the main loss mechanism. Three different values of the two-body scattering length have relevance: $a_{-}$, which characterizes the threshold where the trimer disappears into the three-atom continuum; $a_{*}$, which characterizes the threshold where the trimer disappears into the atom-dimer continuum; and $a_{+}$, which characterizes a minimum in the recombination rate. Discrepancies are found between the zero-range theoretical predictions and experimental determinations of these three quantities [11-14]. In this respect, we would like to see if the quantities obtained from the calculations using finite-range potentials are in better agreement with the experimental predictions.

The paper is organized as follows. In the next section, the two-body and three-body potential models are introduced and the results for the first two energy levels are discussed. The main results of this work are given in Sec. III, which is divided into four sections dedicated to analyze the elastic and breakup cross sections, the recombination and dissociation rates, a comparison of the results to experimental data, and nucleon-deuteron scattering above threshold. The conclusions are given in the last section.

\section{THE THREE-BOSON SYSTEM}

In this section, we study universal aspects of a three-boson system by taking the three-helium system as reference. At the two-body level, we consider one of the most commonly used He-He potentials, i.e., the LM2M2 interaction [7], which is taken as the reference interaction. In particular, in order to explore the $\left(a^{-1}, \kappa\right)$ plane $\left(\kappa=\operatorname{sgn}(E)\left[|E| /\left(\hbar^{2} / m\right)\right]^{1 / 2}\right.$, with $E$ the energy level), we modify this potential as

$$
V_{\lambda}(r)=\lambda V_{\mathrm{LM} 2 \mathrm{M} 2}(r) .
$$

Examples of this strategy exist in the literature [15-17]. For $\lambda \approx 0.9743$, the interaction is close to the unitary limit $(a \rightarrow$ $\infty)$. For $\lambda=1$, the values predicted by the LM2M2 are recovered: the scattering length $a=189.41 a_{0}$, the two-body energy $E_{2}=-1.303 \mathrm{mK}$, and the effective range $r_{e}=13.845 a_{0}$, with the mass parameter $\hbar^{2} / m=43.281307\left(a_{0}\right)^{2} \mathrm{~K}$.

Following Refs. $[8,17,18]$, we define an attractive two-body Gaussian (TBG) potential

$$
V(r)=V_{0} e^{-r^{2} / r_{0}^{2}},
$$

with range $r_{0}=10 a_{0}$ and strength $V_{0}$ fixed to reproduce the values of $a$ given by $V_{\lambda}(r)$. For example, with the strength $V_{0}=-1.2343566 \mathrm{~K}$, corresponding to $\lambda=1$, the LM2M2 low-energy data are closely reproduced, $E_{2}=-1.303 \mathrm{mK}$, $a=189.42 a_{0}$, and $r_{e}=13.80 a_{0}$.

The use of the TBG potential in the three-atom system produces a ground-state binding energy appreciably deeper than the one calculated with $V_{\lambda}(r)$. For example, at $\lambda=1$, the LM2M2 helium-trimer ground-state binding energy is $E_{3}^{0}=126.4 \mathrm{mK}$, whereas the one obtained using the two-body soft-core potential in Eq. (6) is $151.32 \mathrm{mK}$. To solve this discrepancy, we introduce a repulsive hypercentral three-body (H3B) interaction

$$
W\left(\rho_{123}\right)=W_{0} e^{-\rho_{123}^{2} / \rho_{0}^{2}},
$$

with the strength $W_{0}$ tuned to reproduce the trimer energy $E_{3}^{0}$ obtained with $V_{\lambda}(r)$ for all of the explored values of $\lambda$. Here, $\rho_{123}^{2}=\frac{2}{3}\left(r_{12}^{2}+r_{23}^{2}+r_{31}^{2}\right)$ is the hyperradius of three identical particles and $\rho_{0}$ gives the range of the three-body force. Following Ref. [17], we use $\rho_{0}=r_{0}$. It should be noticed that the description of the three-boson systems using a twobody plus three-body interaction constructed to reproduce the low-energy data is equivalent, up to finite-range corrections, to a description based on EFT at LO (see Ref. [19] and references therein).

By varying $\lambda$ from the unitary limit to $\lambda=1.1$, we obtain a set of values for the ground-state binding energy $E_{3}^{0}$ and first excited state $E_{3}^{1}$ using the TBG and TBG $+\mathrm{H} 3 \mathrm{~B}$ potentials in a broad range of $a$. The results can be compared to the predictions given by the Efimov's binding-energy equations,

$$
E_{3}^{n}+\frac{\hbar^{2}}{m a^{2}}=e^{-2\left(n-n^{*}\right) \pi / s_{0}} \exp \left[\Delta(\xi) / s_{0}\right] \frac{\hbar^{2} \kappa_{*}^{2}}{m},
$$

where $\tan \xi=-\left(m E_{3}^{n} / \hbar^{2}\right)^{1 / 2} a$ and the function $\Delta(\xi)$ can be found in [3]. Fixing $n^{*}=1$, the three-body parameter $\kappa_{*}$ is determined by calculating $E_{3}^{1}$ at the unitary limit; we obtain $\kappa_{*}=2.119 \times 10^{-3} a_{0}^{-1}$ and $\kappa_{*}=1.899 \times 10^{-3} a_{0}^{-1}$ for the $\mathrm{TBG}$ and $\mathrm{TBG}+\mathrm{H} 3 \mathrm{~B}$, respectively.

It has been shown in Ref. [8] that in order to be in accord with the numerical results obtained solving a finite-range potential, the universal relation given by Eq. (8) must be modified in the following way:

$$
\begin{aligned}
E_{3}^{n} / E_{2} & =\tan ^{2} \xi, \\
\kappa_{*} a & =e^{\left(n-n^{*}\right) \pi / s_{0}} \exp \left[-\Delta(\xi) / 2 s_{0}\right] / \cos \xi-\Gamma_{n},
\end{aligned}
$$

where the finite-range nature of the interaction has been taken into account by the substitution $\hbar^{2} / m a^{2} \rightarrow E_{2}$, and by the shift $\Gamma_{n}$. In Fig. 1, we collect our numerical results for the ratios $E_{3}^{0} / E_{2}$ and $E_{3}^{1} / E_{2}$ as a function of $\kappa_{*} a$ for the TBG potential (circles) and of the $\mathrm{TBG}+\mathrm{H} 3 \mathrm{~B}$ potential (squares). In the upper panel of Fig. 1, we report the calculations for the ground state. The dashed line corresponds to Eq. (9) without shift, while the solid line, which fits our numerical results, has $\Gamma_{0} \simeq 3 \times 10^{-2}$. In the lower panel of Fig. 1, we report the calculations for the excited state. As above, the dashed line corresponds to Eq. (9) without shift, while the solid line has a finite shift, $\Gamma_{1} \simeq 4 \times 10^{-2}$.

A more accurate analysis of the numerical results reveals that the shift depends on $\kappa_{*}$ [8]; in the first approximation, for the excited state, we can write $\Gamma_{1} \simeq \kappa_{*} r_{*}$ with $r_{*} \approx 21 a_{0}$. From Eq. (9), we can extract $a_{*}^{1}$, which is the scattering length at which the excited state $E_{3}^{1}$ disappears into the atom-dimer 

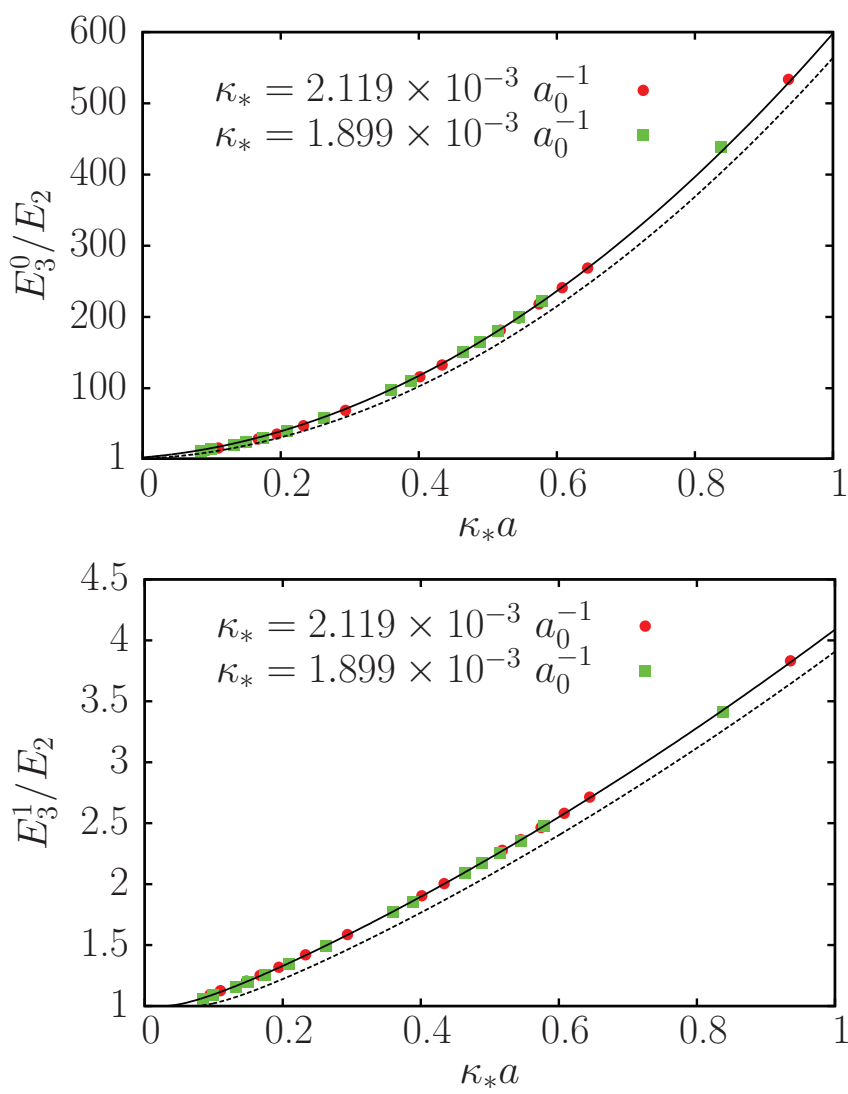

FIG. 1. (Color online) Ratio between the energy of the ground (upper panel) and first excited (lower panel) state of the trimer and the dimer binding energy as a function of $\kappa_{*} a$. The dashed line is the universal prediction of the Efimov law given by Eq. (9) without shift $\left(\Gamma_{n}=0\right)$, while the solid line is the translated universal curve. The circles and squares are the calculations using the TBG and $\mathrm{TBG}+\mathrm{H} 3 \mathrm{~B}$ potentials, respectively.

continuum. This happens when $E_{3}^{1} / E_{2}=1$ or the angle $\xi=$ $-\pi / 4$. Using the very accurate result, $\Delta(-\pi / 4)=6.0273$, given in Ref. [3], we obtain the relation

$$
\kappa_{*} a_{*}^{1}=0.07076-\Gamma_{1},
$$

which in our case gives $a_{*}^{1} \approx 14.5 a_{0}$ and $a_{*}^{1} \approx 16.2 a_{0}$ for the $\mathrm{TBG}$ and $\mathrm{TBG}+\mathrm{H} 3 \mathrm{~B}$ potentials, respectively. Moreover, dividing the above relation by $\kappa_{*}$, we can write $r_{*}=a_{*, \mathrm{zr}}^{1}-a_{*}^{1}$, where we have introduced the universal zero-range scattering length $a_{*, \mathrm{zr}}^{1}=0.07076 / \kappa_{*}$. From this relation, we can interpret the shift, in units of $\kappa_{*}^{-1}$, as the difference between the zero-range and the finite-range predictions for $a_{*}$. In addition, the relation applied to the $n=1$ branch can be extended to the different $n$ branches.

\section{ATOM-DIMER SCATTERING ABOVE THE BREAKUP THRESHOLD}

In Ref. [8], the universal character of the atom-dimer scattering below the breakup threshold has been discussed in terms of the potential model introduced in the previous section. It has been shown that when finite-range interactions are used, the universal formula of the atom-dimer scattering length given in Eq. (1) can be modified as

$$
a_{\mathrm{AD}} / a_{B}=d_{1}+d_{2} \tan \left[s_{0} \ln \left(\kappa_{*} a+\Gamma_{*}\right)+d_{3}\right],
$$

where $a_{B}$ is defined from the relation $E_{2}=\hbar^{2} / m a_{B}^{2}$, and $\Gamma_{*} \simeq$ $\Gamma_{1}$ is the shift for the atom-dimer scattering

Moreover, the effective-range function of Eq. (2) can be adapted to describe finite-range interactions too. In particular, it is given by

$$
k a_{B} \cot \delta=c_{1}(k a)+c_{2}(k a) \cot \left[s_{0} \ln \left(\kappa_{*} a+\Gamma_{\mathrm{e}}\right)+\phi(k a)\right],
$$

with the effective-range shift $\Gamma_{e} \simeq 4 \times 10^{-2}$ [8]. This modified equation agrees with the numerical calculations of Ref. [8], which have been performed by using the potential models of the previous section and the hyperspherical harmonic $(\mathrm{HH})$ method in conjunction with the Kohn variational principle [20], for a wide range of values of $\kappa_{*} a$ varying from 0.26 to 0.94 . In this range, the effective-range function presents different patterns as a function of the energy. For the lowest values of $\kappa_{*} a$, it was almost linear, whereas when increasing $\kappa_{*} a$, a pole structure appeared (see Fig. 4 of Ref. [8]). In addition, it was shown that around the value $\kappa_{*} a \approx 0.54$, the structure of the effective-range function coincides with the one describing neutron-deuteron scattering. In this way, a confirmation of the universal character of Eqs. (11) and (12) has been done for systems with very different typical lengths.

The fact that $\Gamma_{e} \approx \Gamma_{*} \approx \Gamma_{1}$ can be understood noticing that, for the values of $a$ considered, $E_{3}^{1}$ is the only excited state of the three-boson system. Probably, for larger values of $a$, when a second excited state appears, a different shift has to be considered. The analysis of atom-dimer scattering for larger values of $a$ is a numerically difficult task and remains outside the scope of the present work. From Eq. (11), it is possible to extract the value of $a_{*}^{1}$ by equating the argument of the tangent to $-\pi / 2$. Using the value of $d_{3}=1.100$ given in Ref. [8], the results are $a_{*}^{1} \approx 14.3 a_{0}$ and $a_{*}^{1} \approx 16.0 a_{0}$ for the $\mathrm{TBG}$ and $\mathrm{TBG}+\mathrm{H} 3 \mathrm{~B}$ potentials, respectively, in complete agreement with those obtained using Eq. (9). These values can be used to evaluate the ratio $a_{*}^{1} / a_{-}^{0}$ that appears frequently in the literature from measurements on trapped ultracold atoms $[11,12,14]$. The universal zero-range theory predicts $a_{*}^{1} / a_{-}^{0} \approx$ -1.06 , whereas using the values $a_{-}^{0} \approx-43.3 a_{0}$ and $a_{-}^{0} \approx$ $-48.1 a_{0}$ for the TBG and TBG $+\mathrm{H} 3 \mathrm{~B}$ potentials, respectively, given in Ref. [17], we obtain $a_{*}^{1} / a_{-}^{0} \approx-0.32$ in both cases. An analysis of the present result in comparison to those given by different experimental groups is given in Sec. III C.

\section{A. Elastic and breakup cross sections}

The universal character of Eqs. (11) and (12) has been deeply studied in Ref. [8] in a large range of $a$ values. Here we extend the analysis of atom-dimer scattering to energies above the breakup threshold. In particular, we study the universal form predicted by Petrov [6] for the recombination rate at threshold. To this aim, we make use of the adiabatic expansion method as discussed in Refs. [21,22] for energies below the dimer breakup threshold and recently extended to energies above that threshold [23]. As it is well known, the adiabatic expansion method is a very powerful method used to describe bound states [24]. However, the extension to describe 
scattering states encountered some difficulties, in particular in the case of atom-dimer elastic scattering. The problem arose from the difference between the set of coordinates in which the process has a natural asymptotic description (the usual Jacobi coordinates) and the expansion in terms of hyperradial functions which produced a low rate of convergence. In order to circumvent the problem, two integral relations have been derived in Ref. [25] and already applied several times in the literature $[18,21,26]$. Essentially, the method establishes that the scattering matrix is obtained from the following two matrices:

$$
\begin{gathered}
B_{i j}=\frac{2 m}{\hbar^{2}}\left\langle\Psi_{i}^{t}|\hat{\mathcal{H}}-E| F_{j}\right\rangle, \\
A_{i j}=-\frac{2 m}{\hbar^{2}}\left\langle\Psi_{i}^{t}|\hat{\mathcal{H}}-E| G_{j}\right\rangle,
\end{gathered}
$$

where the indexes $i, j$ label the ingoing and outgoing channels (either elastic or inelastic), $\Psi_{i}^{t}$ is the solution of the adiabatic equations at a given energy $E$, and $F_{j}, G_{j}$ are the ingoing and outgoing solutions of the free Schrödinger equation $(T-$ $E) F, G=0$ (see Ref. [23] for details). The $\mathcal{S}$ matrix is then given by the product $A^{-1} B$. The integral relations have a shortrange character, and the problem mentioned above of the mismatch of the coordinates has no consequences once the internal part of the scattering wave function is properly described.

For energies above the dimer breakup threshold, the unitarity of the $\mathcal{S}$ matrix implies that given an incoming channel, for instance, channel $1(1+2$ channel $)$, we have

$$
\sum_{n=2}^{\infty}\left|\mathcal{S}_{1 n}\right|^{2}=1-\left|\mathcal{S}_{11}\right|^{2},
$$

which means that computation of the elastic term $\mathcal{S}_{11}$ amounts to computation of the infinite summation of the $\left|\mathcal{S}_{1 n}\right|^{2}$ terms $(n>1)$ corresponding to the breakup channels. The complex value of $\mathcal{S}_{11}$ can be written in terms of a complex phase shift $\delta$ as

$$
\mathcal{S}_{11}=e^{2 i \delta}=e^{-2 \operatorname{Im}(\delta)} e^{2 i \operatorname{Re}(\delta)}=\left|\mathcal{S}_{11}\right| e^{2 i \operatorname{Re}(\delta)} .
$$

The value of $\left|\mathcal{S}_{11}\right|^{2}$ gives the probability of elastic atom-dimer scattering, and $\left|\mathcal{S}_{11}\right|$ is usually referred to as the inelasticity parameter (for example, denoted by $\eta$ in [27-30]). Obviously, the closer the inelasticity to 1 , the more elastic the reaction. In fact, for energies below the breakup threshold, the phase shift is real and $\left|\mathcal{S}_{11}\right|=1$.

The convergence pattern of the method has been studied in Ref. [23], where the inelasticity $\left(\left|\mathcal{S}_{11}\right|\right)$ and the real part of the phase shift $[\operatorname{Re}(\delta)]$ have been calculated at selected energies for increasing values of $K_{\max }$ (grand-angular quantum number associated to the last adiabatic term included in the expansion of the scattering wave function). The conclusion was that the use of the integral relations produces a pattern of convergence similar to a bound-state calculation. For example, a $K_{\max }$ value of around 12 is enough to get a rather well-converged inelasticity, while $\operatorname{Re}(\delta)$ requires a few more adiabatic terms in order to reach convergence.

To make contact with the results in Ref. [8], in Fig. 2 we show the effective-range function of the TBG potential corresponding to $\kappa_{*} a=0.36$ (upper panel) and $\kappa_{*} a=0.56$ (lower panel) for a wide range of energies. For energies below the dimer threshold, the red circles are the results of Ref. [8],
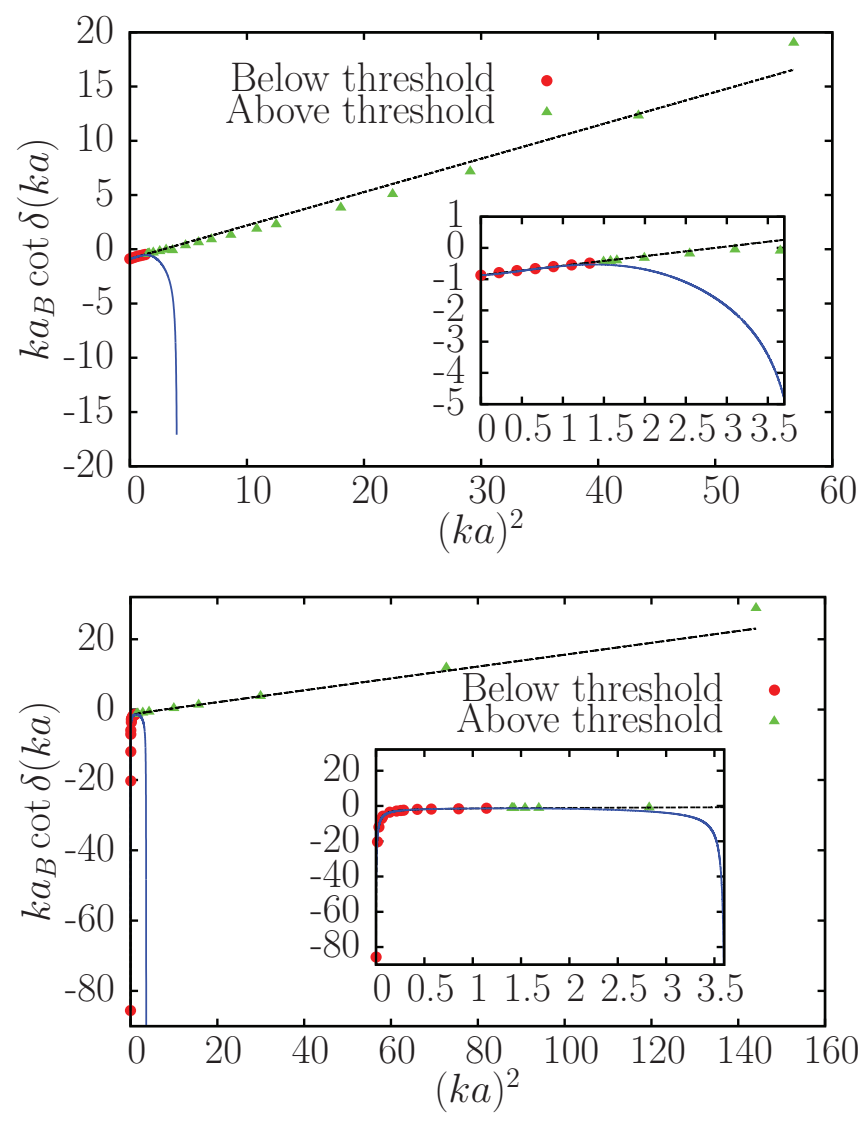

FIG. 2. (Color online) The effective-range function as a function of $(k a)^{2}$ for two different values of $\kappa_{*} a$; in the upper panel, $\kappa_{*} a=0.36$, and, in the lower panel, $\kappa_{*} a=0.56$. The dimer threshold corresponds to $(k a)^{2} \approx 4 / 3$. The (red) circles are the calculations below the dimer threshold of Ref. [8]. The (green) triangles are the present calculations. The solid line is Eq. (12), whereas the dashed line is an effective-range parametrization (see text).

whereas for energies above the dimer threshold, our results obtained using $\operatorname{Re}(\delta)$ in the definition of the effective-range function are given by the green triangles. The solid line has been obtained applying Eq. (12), and the dashed line is the effective-range parametrization of the low-energy points; in the upper panel, the effective range is $k \cot \delta \approx-1 / a_{\mathrm{AD}}+$ $1 / 2 r_{\text {eff }} k^{2}$, with $r_{\text {eff }}$ used as a parameter. In the lower panel, a pole structure dominates the low-energy behavior of the effective-range function, which is better parametrized by $k \cot \delta \approx\left(-1 / a_{\mathrm{AD}}+1 / 2 r_{\text {eff }} k^{2}-P r_{\text {eff }}^{3} k^{4}\right) /\left(1+k^{2} / k_{0}^{2}\right)$; in addition to the effective range, we must introduce the shape parameter $P$ and the momentum of the pole $k_{0}$. From the figure, we can observe that the universal form of Eq. (12) does not describe the points above the dimer threshold. On the other hand, the effective-range parametrization remains close to the computed values in a larger range.

Limiting the discussion to the $L=0$ channel, the determination of the elastic matrix element $\mathcal{S}_{11}$ permits the computation of the elastic $\left(\sigma_{e}\right)$ and breakup $\left(\sigma_{b}\right)$ cross sections, which are given by the well-known expressions

$$
\begin{gathered}
\sigma_{e}=\frac{\pi}{k^{2}}\left|1-\mathcal{S}_{11}\right|^{2}, \\
\sigma_{b}=\frac{\pi}{k^{2}}\left(1-\left|\mathcal{S}_{11}\right|^{2}\right),
\end{gathered}
$$




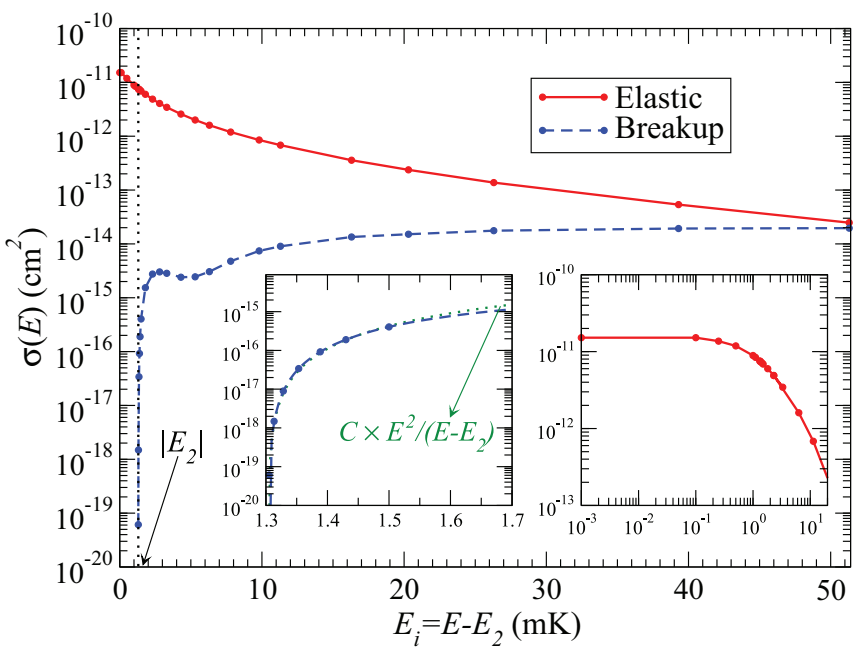

FIG. 3. (Color online) Atom-dimer elastic (solid line) and breakup (dashed line) cross sections as a function of the atom incident energy for a three-helium atom system and the TBG $+\mathrm{H} 3 \mathrm{~B}$ potential. The breakup channel is open at $E_{i}=E_{2}=1.303 \mathrm{mK}$. The zoom shows the behavior close to both thresholds, i.e., $E_{i}=0$ for the elastic case and $E_{i}=E_{2}$ for the breakup case. The circles correspond to actual calculations, while the curves are interpolations.

with $k^{2}=(4 / 3) E_{i} /\left(\hbar^{2} / m\right)$ and $E=E_{i}+E_{2}$, which is the total energy of the process.

Following the method described in [23], we have computed $\sigma_{e}$ and $\sigma_{b}$ for the TBG $+\mathrm{H} 3 \mathrm{~B}$ potential $(\lambda=1$ case $)$. The results are given by the solid and dashed curves in Fig. 3, respectively, where the cross sections are shown for incident energies $E_{i}$ up to $50 \mathrm{mK}$. At this energy, both cross sections have a similar size. In the figure, the corresponding behavior at threshold is zoomed. In the case of the elastic scattering, when $E_{i}$ approaches 0 , the cross section reaches the constant value of $\sigma_{e} \rightarrow 4 \pi a_{\mathrm{AD}}^{2}$. In the case of the breakup cross section, the threshold corresponds to $E=0$ (or $E_{i}=E_{2}$ ), and we have that for $E \rightarrow 0$, the breakup cross section $\sigma_{b}$ behaves as $\sigma_{b} \propto E^{2} /\left(E-E_{2}\right)$ (or, in other words, $\left.1-\left|\mathcal{S}_{11}\right|^{2} \propto E^{2}\right)$.

\section{B. Recombination and dissociation rates}

The breakup cross section described in the previous section is directly related to the dissociation rate, $D_{3}$, for the ${ }^{4} \mathrm{He}_{2}+{ }^{4} \mathrm{He} \rightarrow{ }^{4} \mathrm{He}+{ }^{4} \mathrm{He}+{ }^{4} \mathrm{He}$ process. In particular, for three identical bosons with angular momentum and parity $0^{+}$, it takes the form [31]

$$
D_{3}=\hbar \frac{k}{\mu_{A, d}} \sigma_{b}=\frac{\hbar \pi}{\mu_{A, d} k}\left(1-\left|\mathcal{S}_{11}\right|^{2}\right),
$$

where $\mu_{A, d}=(2 / 3) m$ is the atom-dimer reduced mass and $m$ is the mass of the atom.

Also, making use of the detailed balance principle, it is possible to relate $\sigma_{b}$ to the cross section corresponding to the inverse process ${ }^{4} \mathrm{He}+{ }^{4} \mathrm{He}+{ }^{4} \mathrm{He} \rightarrow{ }^{4} \mathrm{He}_{2}+{ }^{4} \mathrm{He}$. This permits one to obtain the recombination rate $K_{3}$ for such process in terms of the inelasticity parameter. In particular, again for three identical bosons with angular momentum and parity $0^{+}, K_{3}$
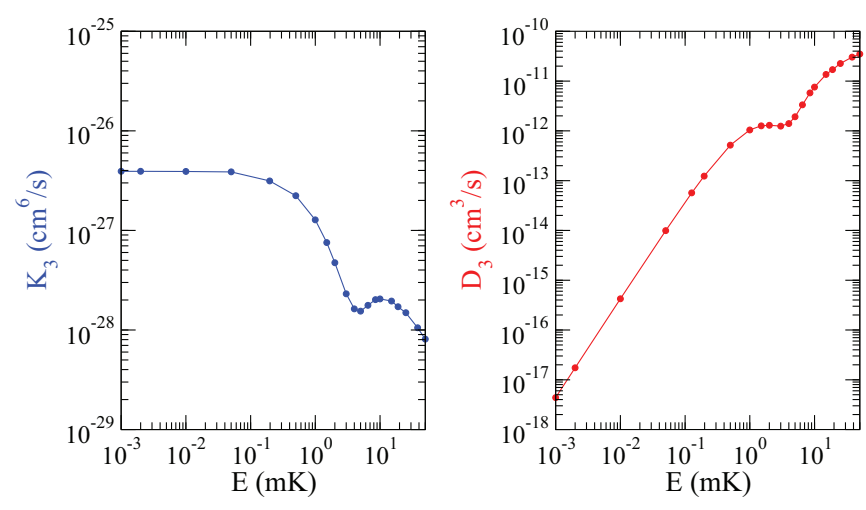

FIG. 4. (Color online) The recombination rate $K_{3}$ and the dissociation rate $D_{3}$ for three-helium atoms with the TBG $+\mathrm{H} 3 \mathrm{~B}$ potential $(\lambda=1)$ as a function of the three-body energy $E$.

takes the form [31]

$$
K_{3}=3 ! \frac{32 \hbar \pi^{2}}{\mu k_{3}^{4}}\left(1-\left|\mathcal{S}_{11}\right|^{2}\right),
$$

where $\mu^{2}=m^{2} / 3$ and $k_{3}^{2}=2 E /\left(\hbar^{2} / \mu\right)$.

At low energies, $K_{3}$ is proportional to $E^{K_{m}}$, as demonstrated in Ref. [32] ( $K_{m}$ is the smallest grand-angular quantum number associated with the continuum adiabatic channels). In the present case, $K_{m}=0$ and $K_{3}$ is almost constant as $E \rightarrow 0$. In the case of $D_{3}$, its low-energy behavior follows the $E^{K_{m}+2}$ rule derived in Ref. [32]. These behaviors can be seen in Fig. 4 in which $K_{3}$ and $D_{3}$ are displayed as a function of the three-body energy $E$ for the TBG $+\mathrm{H} 3 \mathrm{~B}$ calculation $(\lambda=1$ case). This low-energy behavior can also be deduced from the behavior of $\sigma_{b}$ close to threshold shown in the zoom of Fig. 3, and in particular from the fact that when the total energy goes to zero, we have that $1-\left|\mathcal{S}_{11}\right|^{2} \propto k_{3}^{4} \propto E^{2}$.

The recombination rate at threshold can be defined as $K_{3}=$ $\alpha\left(\hbar a^{4} / m\right)$. In Ref. [6], Petrov derived a log-periodic function for $\alpha$ given in Eq. (3), whose simplified version is given by Eq. (4). In order to use this formula to describe our numerical results, which are obtained using finite-range potentials, we introduce the following modification in the definition of $K_{3}$ :

$$
\begin{aligned}
K_{3}= & \frac{128 \pi^{2}(4 \pi-3 \sqrt{3})}{\sinh ^{2}\left(\pi s_{0}\right)+\cosh ^{2}\left(\pi s_{0}\right) \cot ^{2}\left[s_{0} \ln \left(\kappa_{*} a+\Gamma_{+}\right)+\gamma\right]} \\
& \times \frac{\hbar a_{B}^{4}}{m},
\end{aligned}
$$

with the simplified form

$$
K_{3} \approx 67.1 \sin ^{2}\left[s_{0} \ln \left(\kappa_{*} a+\Gamma_{+}\right)+\gamma\right]\left(\hbar a_{B}^{4} / m\right),
$$

where, as in the effective-range function, we have replaced $a$ by $a_{B}$ and we have introduced the shift $\Gamma_{+}$in the variable $\kappa_{*} a$.

Our results are shown in Fig. 5 as circles (TBG potential) and squares $(\mathrm{TBG}+\mathrm{H} 3 \mathrm{~B}$ potential). The dashed line represents the universal function of Eq. (3) with the value $\gamma=1.16$ from Ref. [3]. It is interesting to see that the calculated points organize in a curve shifted with respect to the universal curve. The solid line represents Eq. (21), with the same value of $\gamma$ and $\Gamma_{+} \simeq 6 \times 10^{-2}$. By equating the argument of the sine to zero, we can extract $a_{+}^{1}$, which is the value of $a$ at which the recombination rate has a minimum. We obtain the relation

$$
\kappa_{*} a_{+}^{1}=0.31575-\Gamma_{+}
$$




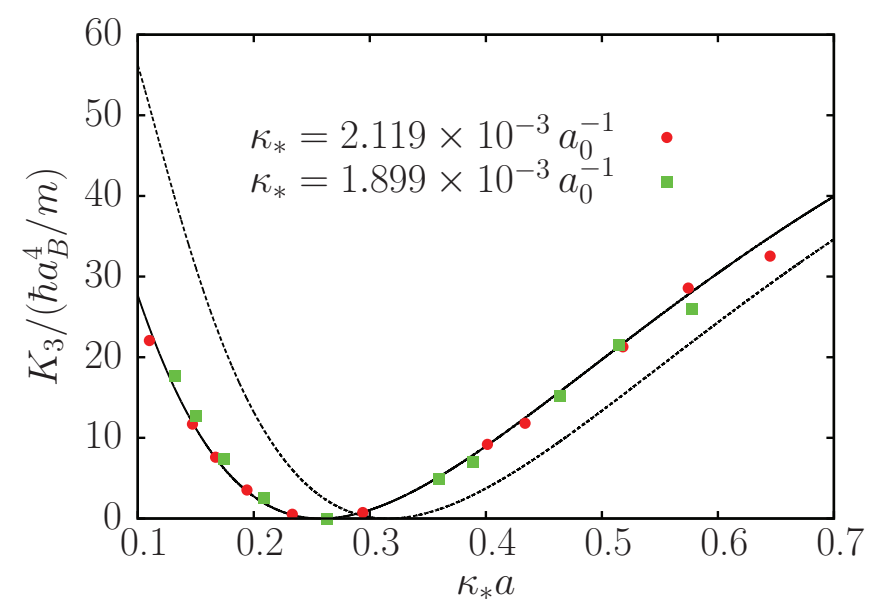

FIG. 5. (Color online) The recombination rate $K_{3}$ at threshold for different values of the product $\kappa_{*} a$. The red circles are the results using the TBG potential, whereas the green squares are the results using the TBG $+\mathrm{H} 3 \mathrm{~B}$ potential. The points have been fitted with Eq. (21), obtaining $\Gamma_{+} \simeq 6 \times 10^{-2}$.

which, in our case, results in $a_{+}^{1} \approx 121 a_{0}$ and $a_{+}^{1} \approx 135 a_{0}$ for the $\mathrm{TBG}$ and $\mathrm{TBG}+\mathrm{H} 3 \mathrm{~B}$ potentials, respectively. With the values $a_{-}^{1} \approx-752 a_{0}$ and $a_{-}^{1} \approx-975 a_{0}$ for the TBG and $\mathrm{TBG}+\mathrm{H} 3 \mathrm{~B}$ potentials, respectively, given in Ref. [17], we obtain $a_{-}^{1} / a_{+}^{1} \approx-6.3$ and $a_{-}^{1} / a_{+}^{1} \approx-7.2$ to be compared to the theoretical prediction of -4.9 . Moreover, defining $\Gamma_{+}=$ $\kappa_{*} r_{+}$and dividing the above relation by $\kappa_{*}$, we can write $r_{+}=$ $a_{+, \mathrm{zr}}^{1}-a_{+}^{1}$, where we have introduced the universal zero-range scattering length $a_{+, \mathrm{zr}}^{1}=0.31575 / \kappa_{*}$. In the present case, we obtain $r_{+} \approx 29 a_{0}$. As in the case of atom-dimer scattering, we can interpret the shift, in units of $\kappa_{*}^{-1}$, as the difference between the zero-range and the finite-range predictions for $a_{+}$. Moreover, the relation applied above to the $n=1$ branch can be extended to the other branches.

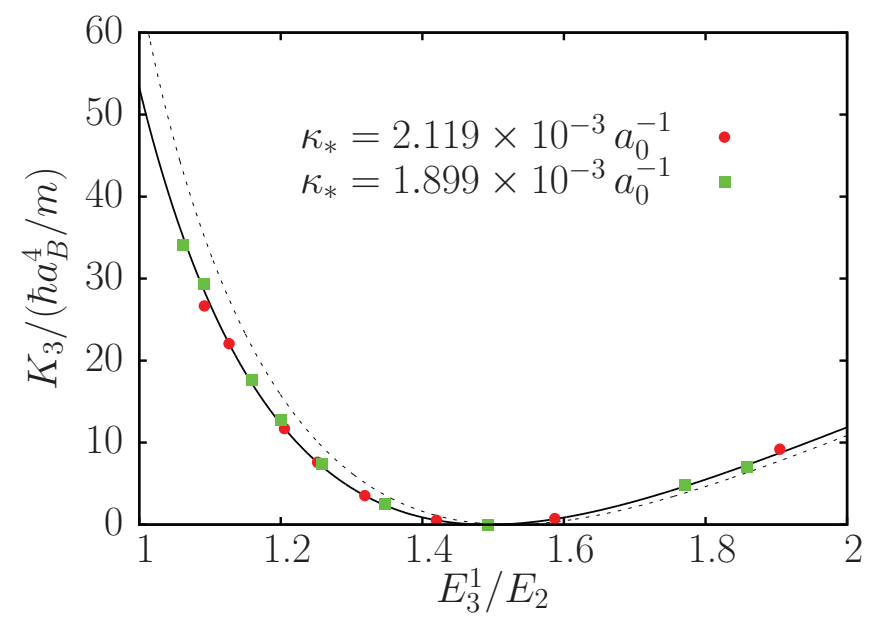

FIG. 6. (Color online) The recombination rate $K_{3}$ at threshold as a function of $E_{3}^{1} / E_{2}$. The red circles are the results using the TBG potential, whereas the green squares are the results using the $\mathrm{TBG}+\mathrm{H} 3 \mathrm{~B}$ potential. The dashed line corresponds to the zero-range theory, while the solid line is the translation of the zero-range curve.
TABLE I. Selected experimental results of the indicated experiments and selected ratios compared to the results of the present work (using the $\mathrm{TBG}+\mathrm{H} 3 \mathrm{~B}$ potential).

\begin{tabular}{lccccc}
\hline \hline & ${ }^{7} \mathrm{Li}[13]$ & ${ }^{7} \mathrm{Li}[14]$ & ${ }^{39} \mathrm{~K}[11]$ & ${ }^{133} \mathrm{Cs}[12]$ & Present work \\
\hline$\ell_{\mathrm{vdW}}\left(a_{0}\right)$ & 32.5 & 32.5 & 64.5 & 101.0 & 5.1 \\
$a_{-}^{0}\left(a_{0}\right)$ & $-241(8)$ & $-280(12)$ & $-690(40)$ & $-872(22)$ & -48.1 \\
$a_{-}^{1}\left(a_{0}\right)$ & & & & & -975 \\
$a_{*}^{1}\left(a_{0}\right)$ & $426(20)$ & $196(4)$ & $930(40)$ & $367(13)$ & 16.0 \\
$a_{+}^{0}\left(a_{0}\right)$ & $88(4)$ & & $224(7)$ & 210 & \\
$a_{+}^{1}\left(a_{0}\right)$ & $1402(100)$ & $1130(120)$ & $5650(900)$ & & 135 \\
$a_{*}^{1} / a_{-}^{0}$ & -1.77 & -0.7 & -1.35 & -0.42 & -0.32 \\
$a_{-}^{0} / a_{+}^{0}$ & -2.7 & & -3.08 & -4.2 & -7.2 \\
$a_{-}^{0} / a_{+}^{1}$ & -0.17 & -0.25 & -0.12 & & -0.35 \\
$a_{-}^{0} / \ell_{\mathrm{vdW}}$ & -7.4 & -8.6 & -10.7 & -8.6 & -9.4 \\
$r_{*} / \ell_{\mathrm{vdW}}$ & -5.5 & 2.9 & -3.4 & 5.3 & 4.1 \\
$r_{+} / \ell_{\mathrm{vdW}}$ & -9.0 & 4.9 & -38.5 & $<0$ & 6.2 \\
\hline \hline
\end{tabular}

Finally, in Fig. 6, we report our results in the form of a Phillips plot, as has usually been done in the literature to study the correlation between different three-body observables (see, for instance, Refs. [3,8,33]). In addition to our numerical data, i.e., circles for $\mathrm{TBG}$ potential and squares for $\mathrm{TBG}+\mathrm{H} 3 \mathrm{~B}$ potential, we report the zero-range calculation without (dashed line) and with (solid line) translation. We observe that the translated curve fits quite well to the numerical data. In fact, Fig. 6 can be understood as the combination of Figs. 1 (lower panel) and 5, where the parameter $\kappa_{*} a$ has been eliminated. We have shown that the finite-range effects manifest as a translation (the shifts) in the parameter $\kappa_{*} a$; see, for instance, Eqs. (9), (11), (12), (21), and (22). Now, the smallness of the shifts justifies the fact that the curve resulting from the elimination of the parameter $\kappa_{*} a$ is itself a translated curve, at least at the first order. If the shifts $\Gamma_{1}$ and $\Gamma_{+}$were the same, then the numerical calculations would had fallen over the zero-range curve without translation. This has been, for instance, demonstrated in Ref. [8] in the case of the atom-dimer scattering length as a function of the excited three-body energy (cf. Fig. 2 of Ref. [8]).

\section{Comparison to the experimental results}

In the following, we compare our numerical results using the $\mathrm{TBG}+\mathrm{H} 3 \mathrm{~B}$ to selected results given in the literature by different groups. We concentrate on the results given for $a_{-}, a_{*}$, and $a_{+}$extracted from resonances observed in trapped ultracold atoms. The value of $a_{-}$is extracted from a maximum in the recombination rate in the negative sector of $a$, whereas $a_{*}$ corresponds to a maximum in an atom-dimer resonance, and $a_{+}$corresponds to a recombination minimum in the positive sector of $a$. In Table I, we collect selected experimental results of these quantities to be compared to the results obtained using the $\mathrm{TBG}+\mathrm{H} 3 \mathrm{~B}$ potential given in the last column. As the numerical results, the measured ratios do not completely agree with the zero-range universal theory. This disagreement can be traced back to the shift introduced in the zero-range theory produced by the finite-range nature of the potential. This shift, which is not universal, produces 
differences between the different atomic species. However, in all cases, the ratio $a_{-}^{0} / \ell_{\mathrm{vdW}} \approx-9$, where $\ell_{\mathrm{vdW}}$ is the natural-atomic length given by the van der Waals ( $\mathrm{vdW}$ ) part of atomic potentials, introduces some common behavior, as was recently justified $[34,35]$. Motivated by this fact, we extract the values of $r_{*}$ and $r_{+}$from the measured experiments. To this end, we can define $\kappa_{*}=(22.7)^{-1} 1.56 /\left|a_{-}^{0}\right|$ from the experimental value of $a_{-}^{0}$ to determine $a_{*, \mathrm{zr}}^{1}$ and $a_{+, \mathrm{zr}}^{1}$ and then calculate the two lengths, which, using Eqs. (10) and (23), can be expressed as

$$
\begin{aligned}
& r_{*}=1.032\left|a_{-}^{0}\right|-a_{*}^{1}, \\
& r_{+}=4.605\left|a_{-}^{0}\right|-a_{+}^{1} .
\end{aligned}
$$

The results in units of $\ell_{\mathrm{vdW}}$ are given in the last two rows of the table. As general trends, we can observe that the absolute value of the two lengths is of the order of $5 \ell_{\mathrm{vdW}}$ (here, potassium is the exception with a rather bigger value of $\left.r_{+}\right)$and $\left|r_{+}\right|$is slightly bigger than $\left|r_{*}\right|$, but the values can be both positives and negatives. The results of the present work agree in order of magnitude with the experimental data, while the signs of both $r_{+}$and $r_{*}$ are probably related to the details of the specific Feshbach resonances. In the presented work, the resonance has been explored by changing the strength of the potential [see Eq. (5)], which is a way to simulate a broad resonance but not a narrow one, and the values of $r_{+}$and $r_{*}$ that we obtain are positives.

Though this analysis is not conclusive, the results show that the shifted formulas can be adapted to describe the experimental results after the determination of $r_{*}$ and $r_{+}$. Even if several efforts have been produced to include finite-range corrections in the interpretation of experimental data [36-41], our results suggest that new experimental as well as theoretical efforts are in order to clarify these kinds of corrections.

\section{The nucleon-deuteron case}

To test the universal description of the recombination rate encoded in the threshold behavior of the $\mathcal{S}$ matrix, we study a system which differs by an order of magnitude on length and energy scales with respect to three-helium atoms. In particular, we shall consider the case of neutron-deuteron $(n-d)$ scattering.

An initial analysis for energies below the deuteron breakup threshold has been done in Ref. [8], where it has been shown that the universal formula of Eq. (12) describes this process quantitatively with $\kappa_{*} a+\Gamma_{*} \approx 0.578$ and $a \approx 4.07 \mathrm{fm}$. These values have been estimated from the pole energy of $E_{p}=$ $-160 \mathrm{keV}$ and the doublet $n$ - $d$ scattering length given in Ref. [42]. In this reference, calculations of $n$ - $d$ scattering have been done using the $s$-wave spin-dependent potential of the Malfliet and Tjon (MT) I-III model defined as

$$
\begin{aligned}
& V_{t}(r)=\frac{1}{r}\left(-626.885 e^{-1.55 r}+1438.72 e^{-3.11 r}\right), \\
& V_{s}(r)=\frac{1}{r}\left(-513.968 e^{-1.55 r}+1438.72 e^{-3.11 r}\right),
\end{aligned}
$$

where $s$ and $t$ are the singlet and triplet spin states, respectively, $r$ is given in $\mathrm{fm}$, and the potential is given in MeV. With

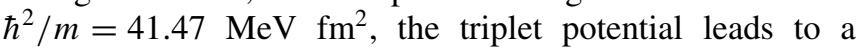
binding energy for the deuteron of $E_{2}=2.2307 \mathrm{MeV}$. The
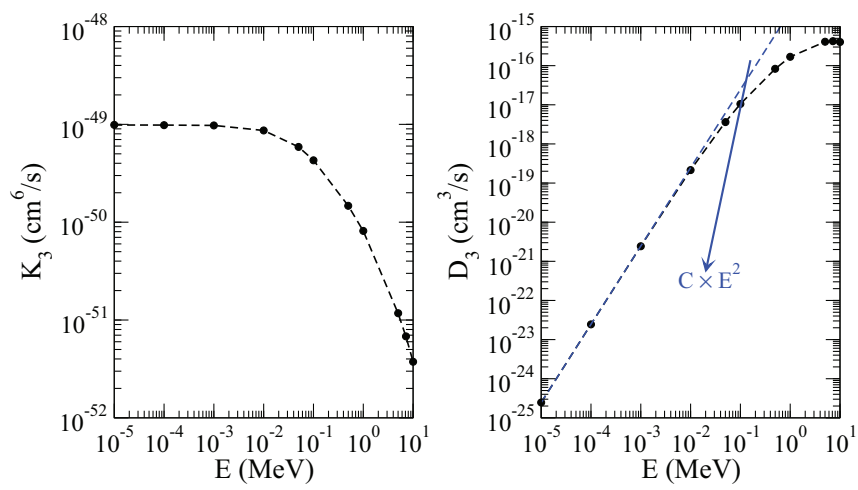

FIG. 7. (Color online) The recombination rate $K_{3}$ and the dissociation rate $D_{3}$ for the $1 / 2^{+}$state in the nucleon-deuteron system as a function of the energy.

predictions for the singlet and triplet scattering lengths are $a_{s}=-23.583 \mathrm{fm}$ and $a_{t}=5.513 \mathrm{fm}$, respectively, which is very close to the experimental results. The scattering lengths are substantially bigger than the effective range of the system, $r_{s}>2 \mathrm{fm}$, allowing an analysis in terms of the universal formulas.

Using the potentials above and the procedure described in [23], we have calculated the corresponding recombination and the dissociation rates, which are shown in Fig. 7 as a function of the three-body energy $E$. The figure shows the $J=1 / 2^{+}$ state, which is the only one having a $K_{m}=0$ contribution, being then the most important one at low energies. When the threshold is approached $(E \rightarrow 0)$, the rates have the same behavior as in the atomic case, i.e., $K_{3}$ goes to a constant and $D_{3}$ goes to zero as $E^{2}$ (as shown by the dashed line in the right part of the figure). At threshold, we obtain $K_{3}=9.85 \times$ $10^{-49} \mathrm{~cm}^{6} / \mathrm{s}$.

It is possible to analyze the behavior of $K_{3}$ at threshold in the context of the universal function of Eq. (21). However, it should be noticed that we cannot simply replace the value of the triplet scattering length, since both channels, i.e., the spin triplet and the spin singlet, contribute to the process. To determine the value of the scattering length to be used, let us have a look into the $J=1 / 2^{+}$scattering wave function $\Psi_{\text {nd }}$, which is usually expanded in terms of a complete set of adiabatic functions as [23]

$$
\Psi_{\text {nd }}(\rho, \Omega)=\frac{1}{\rho^{5 / 2}} \sum_{n=1}^{\infty} f_{n}(\rho) \Phi_{n}(\rho, \Omega),
$$

where $\rho, \Omega$ are the hyperradial and hyperspherical coordinates and the $\Phi_{n}(\rho, \Omega)$ functions are, in fact, the eigenfunctions of the angular part of the Schrödinger (or Faddeev) equations. The angular eigenfunctions are typically expanded in terms of the hyperspherical harmonics, such that for our case with total angular momentum $L=0, \Phi_{n}(\rho, \Omega)$ is written as

$$
\begin{aligned}
\Phi_{n}(\rho, \Omega)= & \sum_{K}\left[C_{K, 0}(\rho) Y_{K}(\Omega)\left|s_{x}=0\right\rangle\right. \\
& \left.+C_{K, 1}(\rho) Y_{K}(\Omega)\left|s_{x}=1\right\rangle\right],
\end{aligned}
$$

where $Y_{K}$ are $\mathrm{HH}$ functions, $C_{K, s_{x}}(\rho)$ are the coefficients in the expansion, and we have made explicit the contributions from the possible spin states $s_{x}=0,1$ of two nucleons, which, after 


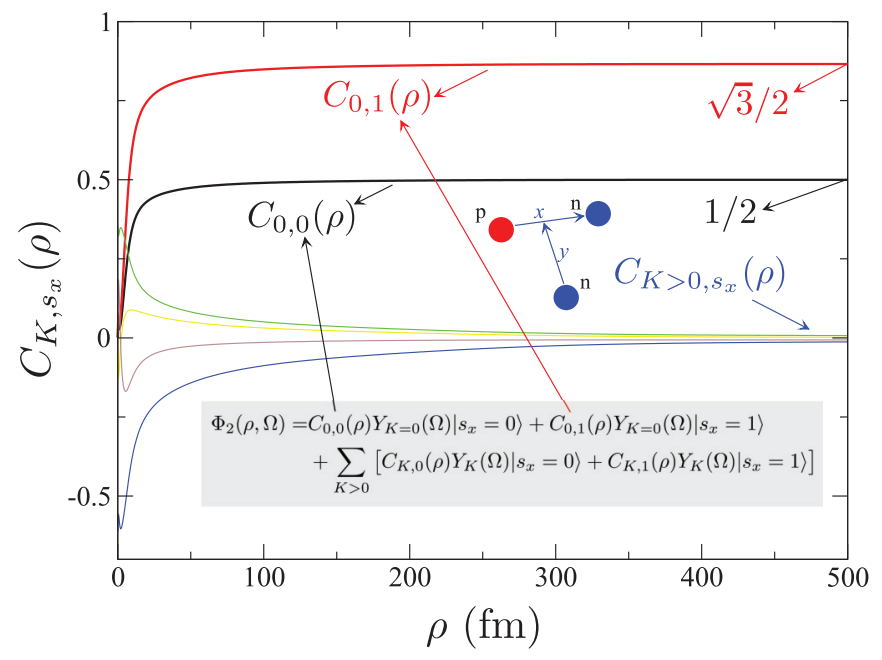

FIG. 8. (Color online) The coefficients of the scattering wave function as a function of the hyperradius $\rho$. The picture show the Jacobi set of coordinates used, whereas the shadow box indicates the two coefficients surviving at long distances.

coupled to the spin of the third nucleon, give the total angular momentum of $1 / 2$.

The $n=1$ term describes asymptotically the elastic channel and $\Phi_{1}(\rho, \Omega)$ is proportional to the deuteron wave function. The infinitely many remaining adiabatic channels describe the breakup process [23]. Among them, the only one that asymptotically contains contributions from hyperspherical harmonics with grand-angular momentum $K=0$ is the lowest adiabatic breakup channel (the $n=2$ term). This is the channel responsible for the behavior of the reaction rates at threshold.

In Fig. 8, the coefficients of the $\Phi_{2}(\rho, \Omega)$ wave function are shown as a function of the hyperradius. All of the coefficients with $K>0$ go to zero as $\rho \rightarrow \infty$, but for $K=0$, the two coefficients $C_{0,0}(\rho)$ and $C_{0,1}(\rho)$ go to $1 / 2$ and $\sqrt{3} / 2$, respectively. In the figure, the Jacobi coordinates have been depicted indicating, by $x$, the $n-p$ relative distance and, by $y$, the relative distance of the third nucleon to the $n-p$ center of mass, so $s_{x}=0,1$ is the $n-p$ spin. The shadow box shows explicitly the two surviving terms when $\rho$ increases.

This analysis implies that $K_{3}$ receives contribution from the singlet and triplet channel properly weighted. Therefore, in the universal formula, the scattering length $a$ has to be considered as an effective scattering length $a=12.68 \mathrm{fm}$, obtained from the average $a^{2}=\left(3 a_{t}^{2}+a_{s}^{2}\right) / 4$. Moreover, the value of $a_{B}$ results in $12.37 \mathrm{fm}$ if, in the average, $a_{t}$ is replaced by $\hbar / \sqrt{m E_{2}}$. With the values for $a, a_{B}$ and the product $\kappa_{*} a+$ $\Gamma_{+} \approx 1.8$ (obtained from the $n-d$ value of 0.578 multiplied to the ratio of $a / a_{t}$ ), we obtain from Eq. (21) the recombination rate value of $K_{3}=9.5 \times 10^{-49} \mathrm{~cm}^{6} / \mathrm{s}$, which is very close to the computed value. This qualitative analysis can be used as a starting point in the spin analysis of the universal aspects of the recombination rate in light nuclear systems.

\section{CONCLUSIONS}

In the present paper, we have investigated atom-dimer scattering above the breakup threshold using a set of Gaussian potentials constructed to reproduce the low-energy atom-atom system. We have introduced a three-body force in order to reproduce the trimer binding energies given by the LM2M2 potential multiplied by a constant $\lambda$ varied from $\lambda \approx 0.974$ to $\lambda \approx 1.1$ in order to cover a large part of the $\left(a^{-1}, \kappa\right)$ plane. We have studied the total elastic and the total breakup cross sections, as well as the recombination and dissociation rates, $K_{3}$ and $D_{3}$, which are directly related to the latter. We have paid special attention to the behavior of the system close to threshold, and we have investigated the universal behavior of $K_{3}$ for different values of the two-body scattering length $a$. Our aim here was to analyze the differences between the universal zero-range theory which, for $K_{3}$, are given by Eq. (3), and more realistic finite-range calculations. This study started already in Ref. [8], where these kinds of differences have been studied for the first excited level of the three-atom system and in the effective-range function. In that reference, it was found that finite-range results organize in a curve shifted with respect to the zero-range theory. Here we have extended the analysis to the ground-state energy and to the recombination rate. Our main results are given in Fig. 5, where we can observe that the computed values for $K_{3}$ lie on a shifted curve in the variable $\kappa_{*} a$. Additional range corrections come from the replacement of $a$ by $a_{B}$ in the $\hbar a^{4} / m$ factor. This type of correction has been already introduced in the study of $a_{\mathrm{AD}}$ and $k \cot \delta$, as well as in the binding energies $E_{3}^{0}$ and $E_{3}^{1}$ which have been divided by $E_{2}$ instead of $\hbar^{2} / m a^{2}$, as indicated by the zero-range theory.

The fact that the finite-range results lie on a shifted curve in the variable $\kappa_{*} a$ for the binding energies and the low-energy scattering quantities strongly supports this particular type of correction. Following this observation, we have proposed Eqs. (11), (12), and (21) as modifications to the zero-range universal formulas where we have introduced the shifts $\Gamma_{*}, \Gamma_{e}$, and $\Gamma_{+}$. From the calculations, we find that the shift of the first excited $\Gamma_{1} \approx \Gamma_{*} \approx \Gamma_{e}$, whereas $\Gamma_{+}$is slightly larger. We have argued that in the range of $a$ studied, the three-boson system has only one excited state, making the shift of the same order. In the case of the recombination rate, one more channel is open and, in terms of adiabatic potentials, at least two channels have to be considered (see, for example, Sec. 6 of Ref. [3]). As a consequence, a larger amount of potential energy appears, increasing the shift. This intuitive argument can be stated formally; work along this line is in progress.

Most of the work presented here has been focused on the necessity of adapting the universal formulas obtained in a zero-range theory to the physical case of finite-range interactions. Accordingly, the modifications introduced in the three-helium system model similar modifications to be considered in the analysis of the experiments on Efimov resonance in ultracold gases. The analysis of the experimental results given in Sec. III C was directed to see if there is experimental evidence of the lengths $r_{*}$ and $r_{+}$introduced in the parametrization of the shift. We have observed some common behavior of the different species when the lengths are given in units of $\ell_{\mathrm{vdW}}$. This fact encourages this kind of analysis and suggests that more theoretical as well experimental efforts can be made in this direction.

In the last part of this study, we have focused on the study of nucleon-deuteron scattering closely above the breakup 
threshold. Using the spin-dependent MT I-III potential, we have shown that the $K_{3}$ and $D_{3}$ rates have a behavior close to threshold, similar to the one observed in the three-atom systems. We have applied the universal formula to this system using a spin average scattering length and we have seen that it is possible to describe the computed value of $K_{3}$. This qualitative analysis can be considered as a first step in the study of universal aspects of nucleon-deuteron scattering considering the full spin dependence of the system.

\section{ACKNOWLEDGMENTS}

E.G. acknowledges financial support provided by DGI of MINECO (Spain) under Contract No. FIS2011-23565.
[1] V. Efimov, Phys. Lett. B 33, 563 (1970).

[2] V. Efimov, Sov. J. Nucl. Phys. 12, 589 (1971) [Yad. Fiz. 12, 1080 (1970)].

[3] E. Braaten and H.-W. Hammer, Phys. Rep. 428, 259 (2006).

[4] E. Nielsen and J. H. Macek, Phys. Rev. Lett. 83, 1566 (1999).

[5] B. D. Esry, C. H. Greene, and J. P. Burke, Phys. Rev. Lett. 83, 1751 (1999).

[6] D. S. Petrov (unpublished).

[7] R. A. Aziz and M. J. Slaman, J. Chem. Phys. 94, 8047 (1991).

[8] A. Kievsky and M. Gattobigio, Phys. Rev. A 87, 052719 (2013).

[9] A. Deltuva and A. C. Fonseca, Phys. Rev. C 87, 014002 (2013).

[10] M. Yamashita, T. Frederico, and L. Tomio, Phys. Lett. B 670, 49 (2008).

[11] M. Zaccanti, B. Deissler, C. D’Errico, M. Fattori, M. JonaLasinio, S. Müller, G. Roati, M. Inguscio, and G. Modugno, Nat. Phys. 5, 586 (2009); S. Roy, M. Landini, A. Trenkwalder, G. Semeghini, G. Spagnolli, A. Simoni, M. Fattori, M. Inguscio, and G. Modugno, Phys. Rev. Lett. 111, 053202 (2013).

[12] F. Ferlaino, A. Zenesini, M. Berninger, B. Huang, H. C. Nägerl, and R. Grimm, Few-Body Syst. 51, 113 (2011).

[13] P. Dyke, S. E. Pollack, and R. G. Hulet, arXiv:1302.0281 [Phys. Rev. A (to be published)].

[14] O. Machtey, Z. Shotan, N. Gross, and L. Khaykovich, Phys. Rev. Lett. 108, 210406 (2012).

[15] B. D. Esry, C. D. Lin, and C. H. Greene, Phys. Rev. A 54, 394 (1996).

[16] P. Barletta and A. Kievsky, Phys. Rev. A 64, 042514 (2001).

[17] M. Gattobigio, A. Kievsky, and M. Viviani, Phys. Rev. A 86, 042513 (2012).

[18] A. Kievsky, E. Garrido, C. Romero-Redondo, and P. Barletta, Few-Body Syst. 51, 259 (2011).

[19] P. F. Bedaque, H. W. Hammer, and U. v. Kolck, Nucl. Phys. A 646, 444 (1999).

[20] A. Kievsky, Nucl. Phys. A 624, 125 (1997).

[21] A. Kievsky, Few-Body Syst. 50, 69 (2011).

[22] C. Romero-Redondo, E. Garrido, P. Barletta, A. Kievsky, and M. Viviani, Phys. Rev. A 83, 022705 (2011).
[23] E. Garrido, C. Romero-Redondo, A. Kievsky, and M. Viviani, Phys. Rev. A 86, 052709 (2012).

[24] E. Nielsen, D. V. Fedorov, A. S. Jensen, and E. Garrido, Phys. Rep. 347, 373 (2001).

[25] P. Barletta, C. Romero-Redondo, A. Kievsky, M. Viviani, and E. Garrido, Phys. Rev. Lett. 103, 090402 (2009).

[26] K. M. Nollett, Phys. Rev. C 86, 044330 (2012).

[27] J. L. Friar, B. F. Gibson, G. Berthold, W. Glöckle, T. Cornelius, H. Witala, J. Haidenbauer, Y. Koike, G. L. Payne, J. A. Tjon, and W. M. Kloet, Phys. Rev. C 42, 1838 (1990).

[28] J. L. Friar, G. L. Payne, W. Glöckle, D. Hüber, and H. Witała, Phys. Rev. C 51, 2356 (1995).

[29] A. Kievsky, M. Viviani, and S. Rosati, Phys. Rev. C 64, 024002 (2001).

[30] A. Kievsky, S. Rosati, and M. Viviani, Phys. Rev. Lett. 82, 3759 (1999).

[31] H. Suno and B. D. Esry, Phys. Rev. A 78, 062701 (2008).

[32] B. D. Esry, C. H. Greene, and H. Suno, Phys. Rev. A 65, 010705 (2001).

[33] M. T. Yamashita, T. Frederico, L. Tomio, and A. Delfino, Phys. Rev. A 68, 033406 (2003).

[34] J. Wang, J. P. D'Incao, B. D. Esry, and C. H. Greene, Phys. Rev. Lett. 108, 263001 (2012).

[35] P. Naidon, S. Endo, and M. Ueda, arXiv:1208.3912.

[36] T. Frederico, L. Tomio, A. Delfino, and A. E. A. Amorim, Phys. Rev. A 60, R9 (1999).

[37] P. Naidon, E. Hiyama, and M. Ueda, Phys. Rev. A 86, 012502 (2012).

[38] C. Ji, D. R. Phillips, and L. Platter, Europhys. Lett. 92, 13003 (2010).

[39] J. P. D'Incao, C. H. Greene, and B. D. Esry, J. Phys. B 42, 044016 (2009).

[40] M. Thøgersen, D. V. Fedorov, and A. S. Jensen, Phys. Rev. A 78, 020501 (2008).

[41] L. Platter, C. Ji, and D. R. Phillips, Phys. Rev. A 79, 022702 (2009).

[42] C. R. Chen, G. L. Payne, J. L. Friar, and B. F. Gibson, Phys. Rev. C 39, 1261 (1989). 